\title{
Prevalence and antimicrobial susceptibility profile of multidrug-resistant bacteria among intensive care units patients at Ain Shams University Hospitals in Egypt-a retrospective study
}

Noha Alaa Eldin Fahim(1)

\begin{abstract}
Background: The nightmare of the rising numbers of multidrug-resistant organisms (MDROs) requires the implementation of effective stewardship programs. However, this should be preceeded by making available evidence-based knowledge regarding the local antimicrobial resistance pattern, which is fundamental. The aim of the current study is to determine the prevalence of MDRO among different Ain Shams University Hospitals (ASUHs) intensive care units (ICUs) and detect the resistance profile of the common pathogens.

Results: The 1-year records of a total of 1280 pathogens were studied. The highest number of pathogens were isolated from blood cultures (44.84\%), followed by urine (41.41\%) then wound swabs (13.75\%). Gram-negative isolates (57.5\%) were more prevalent than gram-positive ones (31.1\%). The most frequently isolated pathogens were Klebsiella spp. (22.5\%), Escherichia coli (13.4\%), and Coagulase-negative Staphylococci (12.5\%). The highest percentage of resistance among gram-positive organisms was exhibited by penicillin (89.5\%) followed by erythromycin (83.98\%) and then cefoxitin (76.52\%). None of the isolates showed resistance to linezolid and resistance to vancomycin was minimal (2.62\%). Gram-negative isolates exhibited high overall resistance to all used antibiotic classes. The least frequency of resistance was recorded against nitrofurantoin (52.5\%), amikacin (58.01\%), followed by imipenem (59.78\%) and meropenem (61.82\%). All isolates of Pseudomonas and Acinetobacter showed 100\% susceptibility to colistin.

Conclusions: The prevalence of antibiotic resistance in Ain Shams University Hospitals (ASUHs) was high among both gram-negative and gram-positive organisms. This high resistance pattern foreshadows an inevitable catastrophe that requires continuous monitoring and implementation of effective antibiotic stewardship.
\end{abstract}

Keywords: Multidrug resistance; Egypt, Prevalence, ICU, Susceptibility profile

Correspondence: dr_nohaalaa@yahoo.com; dr_nohaalaa@med.asu.edu.eg

Clinical Pathology Department, Faculty of Medicine, Ain Shams University,

Cairo, Egypt

(c) The Author(s). 2021 Open Access This article is licensed under a Creative Commons Attribution 4.0 International License, which permits use, sharing, adaptation, distribution and reproduction in any medium or format, as long as you give appropriate credit to the original author(s) and the source, provide a link to the Creative Commons licence, and indicate if changes were made. The images or other third party material in this article are included in the article's Creative Commons licence, unless indicated otherwise in a credit line to the material. If material is not included in the article's Creative Commons licence and your intended use is not permitted by statutory regulation or exceeds the permitted use, you will need to obtain permission directly from the copyright holder. To view a copy of this licence, visit http://creativecommons.org/licenses/by/4.0/. 


\section{Background}

Antimicrobial resistance is rapidly becoming a global focus of attention, especially with the rising number of microorganisms resistant to available antimicrobials. It encompasses both the gram-positive and gram-negative bacteria, with global prevalence rates of $60 \%$ or more [1].

Multidrug-resistant organisms (MDROs) are described as acquired non-sensitivity to one or more agents in at least three groups of antimicrobials. This kind of resistance essentially predominates in hospitals [2].

The lack of quick proper identification of pathogens especially in patients with critical infection led to broadspectrum antibiotics overuse. As a result of this dilemma, organisms became resistant to all available antimicrobial agents and susceptible only to older, likely more toxic antimicrobials, leaving less effective scanty alternatives [3, 4]. The Centers for Disease Control and Prevention (CDC) declared that worldwide increasing infection rates with resistant pathogens strikingly endanger our healthcare systems creating both negative universal economic effects and a therapeutic challenge for clinicians hence delaying proper antibiotic therapy and increasing mortality rates [5].

To combat this horrifying ascent in antimicrobial resistance, the World Health Organization (WHO) urges healthcare providers to adopt antimicrobial stewardship to decrease the heavy cargo of antibiotic resistance. However, before the implementation of any stewardship program, information on prevalent MDRO and their antimicrobial resistance profile are required [6].

Data about the endemic antimicrobial resistance are generally difficult to find, particularly in countries where antibiotics are easily obtainable over the counter. Although numerous reports demonstrated the incidence and the patterns of resistance of many pathogens, few studies about the endemic antimicrobial resistance profile in developing countries were published [3, 7].

Hence, an evidence-based knowledge regarding the local antimicrobial resistance pattern is fundamental for guiding both antimicrobial treatment and empirical therapy of specific pathogens [8]. This guide is also important for effective antimicrobial stewardship as well as in the design of local and universal research programs [3].

Since the intensive care unit (ICU) patients are more prone to nosocomial infections caused by aggressive pathogens, therefore, the present study aimed to identify and obtain a comprehensive idea about the current situation in Ain Shams University Hospitals (ASUHs) regarding the spectrum of microbes and the antimicrobial resistance pattern of the most prevalent pathogens isolated from variable infection sites of ICU patients in addition to the determination of the prevalence of multiple drug resistance through a 1-year retrospective study.

\section{Methods}

\subsection{Study design}

A record-based 1-year retrospective study-from March 2018 going through February 2019-was conducted at ASUHs one of the largest tertiary hospitals in Egypt with more than 3200-bed capacity.

The records of the pathogenic organisms recovered from different microbiological samples (blood, urine, and wound) of ICU patients sent for routine diagnosis in microbiology laboratory were retrieved from the microbiology laboratory information system at ASUHs and reviewed. Information regarding the identified bacterial isolate, specimen type, and antimicrobial susceptibility was collected and recorded.

\subsection{Microbiological specimens processing and identification of isolated organisms}

Sample processing and identification of the microorganism were performed per the standard operating procedures of our laboratory. All the used media were purchased from (Oxoid, UK).

The samples were cultured on the routinely used microbiological media and incubated for $24 \mathrm{~h}$ at $37{ }^{\circ} \mathrm{C}$. If no growth, the plates were incubated for a total of 48 hours.

The isolated microorganisms' recognition was done according to colony morphology, Gram stain, and standard confirmatory biochemical tests were used.

Gram-positive bacteria were identified via testing the hemolytic activity on blood agar and further identification using different biochemical tests as catalase reaction, slide and tube coagulase tests, culture on DNase agar, bile esculin, in addition to different differentiating antibiotic discs as optochin and bacitracin. For gram-negative bacteria, identification was conducted by biochemical tests such as oxidase, triple sugar iron, motility indole ornithine, citrate, lysine iron arginine, and urease tests. They were further identified by Vitek2 system (Biomerieux, France).

\subsection{Antimicrobial susceptibility}

Antimicrobial susceptibilities of the bacterial isolates by Kirby-Bauer disk diffusion method were performed and interpreted according to the Clinical Laboratory Standards Institute (CLSI) guidelines [9]. The tested antibiotic discs were routinely supplied from Oxoid. Staphylococcus aureus ATCC 25923, Pseudomonas aeruginosa ATCC 27853, and Escherichia coli ATCC 25922 were used as controls for susceptibility testing.

Extended-spectrum beta-lactamase (ESBL) production was detected using the double-disc synergy test by placing amoxicillin/clavulanate disc adjacent to cefotaxime and ceftazidime discs and looking for synergy between the clavulanic acid and the cephalosporin [10]. 
Colistin resistance in both Acinetobacter spp. and Pseudomonas spp. was detected by Vitek2 system.

Methicillin resistance in Staphylococcus species both Staphylococcus aureus (MRSA) and coagulase-negative (MR-CONS) was detected using cefoxitin disc $(30 \mu \mathrm{g})$.

Vancomycin-resistant Enterococci (VRE) were initially screened for using agar dilution method on brain heart infusion agar (BHI) with $6 \mu \mathrm{g} / \mathrm{ml}$ vancomycin and further confirmation for any suspected colonies was accomplished by Vitek 2 system (Biomerieux, France).

\subsection{Multiple drug resistance (MDR)}

MDR isolates were described by the CDC as acquired non-sensitivity to one or more agents in at least three groups of antimicrobials [11].

\subsection{Statistical analysis}

Data were presented as percentages and counts. Statistical analysis was performed using the statistical package for social sciences (SPSS) computer software (Version 25), IBM software, USA. Pearson chi-square test or Fisher's exact test-after checking the applicability conditionswere performed to identify the significant effect of each antibiotic on different isolates. A statistically significant difference was considered at $p$ value $\leq 0.05$.

\section{Results}

3.1 Spectrum of pathogens in different clinical specimens The 1 -year records of a total of 1280 pathogens from different clinical samples were retrieved from the laboratory information system of ASUH during the specified time period. The highest number of pathogens were isolated from blood cultures $(44.84 \%, n=574)$, followed by urine where $(41.41 \%, n=530)$ pathogens were recovered, while the least isolation rate was exhibited by wound swabs $(13.75 \%, n=176)$. Gram-negative isolates $(57.5 \%, n=736)$ were more prevalent compared to gram-positive ones $(31.1 \%, n=399)$. The most frequently isolated pathogens were Klebsiella spp. (22.5\%, $n=288)$, Escherichia coli (E. coli) $(13.4 \%, n=172)$, and Coagulase-negative Staphylococci (CONS) $(12.5 \%, n=161)$, while Proteus, non-hemolytic Streptococci, Enterobacter spp., and others constituted the smallest group among the studied isolates (Fig. 1). Bloodstream infections were caused mainly by CONS $(24.2 \%, n=139 / 574)$ and Klebsiella spp. (23.8\%, $n=137 / 574)$. Candida spp. (22.5\%, $n=126 / 530)$, E. coli (20\%, $n=106 / 530)$, and Klebsiella spp. (19\%, $n=101 / 530)$ were the main incriminated pathogens in urinary tract infections. The frequently isolated pathogens from wounds were Klebsiella spp. $(28.4 \%, n=50 / 176)$, Pseudomonas spp. (17.6\%, $n=31 / 176)$, and Acinetobacter spp. $(15.9 \%, n=28 / 176)$. The distribution of pathogens among the different types of specimens is summarized in Table 1.

\subsection{Antibiotic resistance pattern of isolates recovered from various sites of infection}

The distribution of pathogens and their patterns of resistance are presented in Table 2.

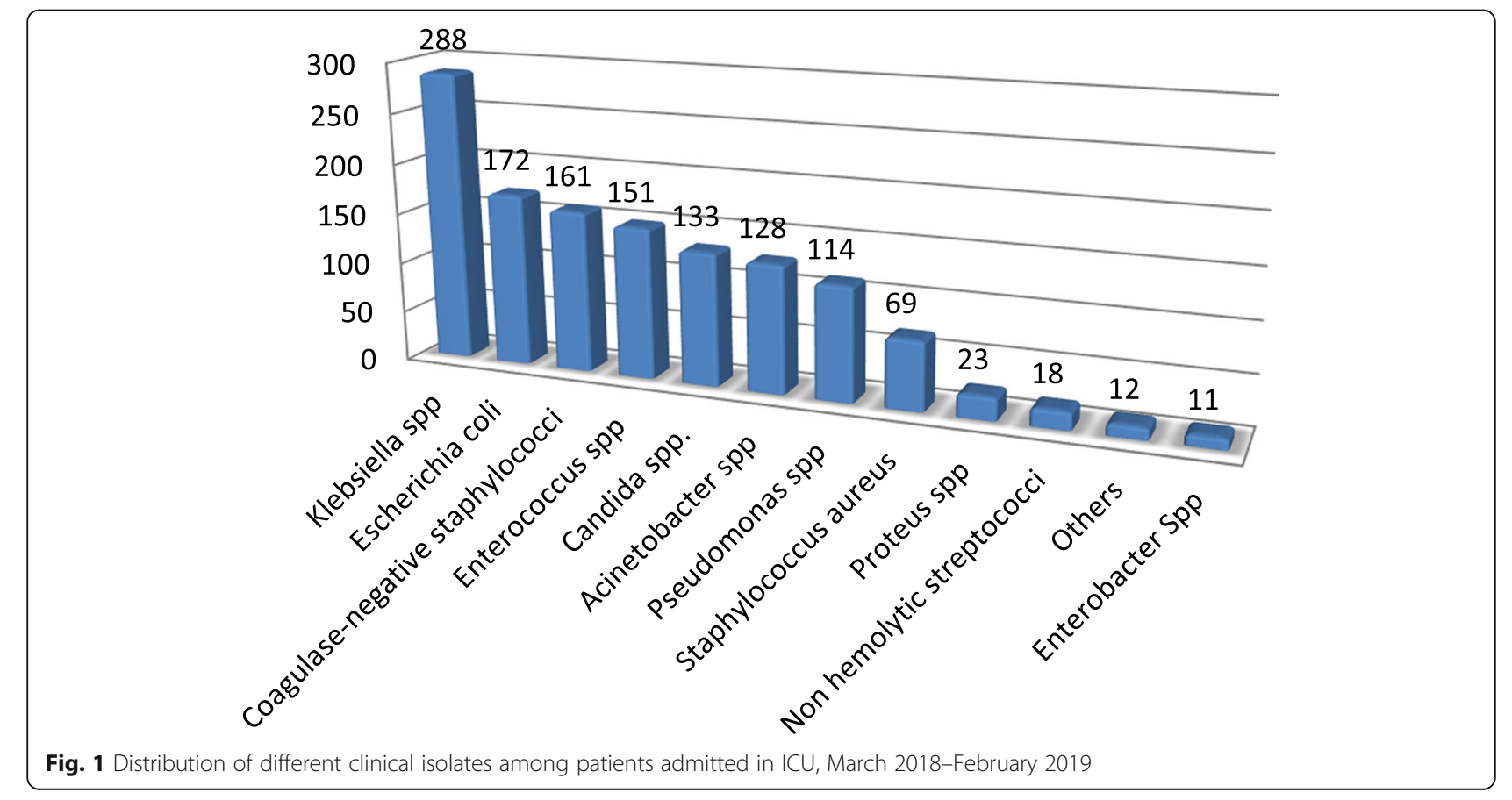


Table 1 Frequency of different pathogens within various clinical specimens collected from ICU patients, Ain Shams University Hospitals, Egypt, March 2018-February 2019

\begin{tabular}{|c|c|c|c|c|}
\hline \multirow[t]{2}{*}{ Name of organism } & \multirow[t]{2}{*}{ Total } & \multicolumn{3}{|l|}{$N(\%)$} \\
\hline & & Urine & Blood & Wound swab \\
\hline Klebsiella spp. & 288 & $101(35.06 \%)$ & $137(47.56 \%)$ & $50(17.38 \%)$ \\
\hline E.coli & 172 & $106(61.62 \%)$ & $48(27.91 \%)$ & $18(10.47 \%)$ \\
\hline Acinetobacter spp. & 128 & $33(25.78 \%)$ & $67(52.34 \%)$ & $28(21.88 \%)$ \\
\hline Pseudomonas spp. & 114 & $49(42.98 \%)$ & $34(29.82 \%)$ & $31(27.20 \%)$ \\
\hline Proteus spp. & 23 & $5(21.73 \%)$ & $6(26.10 \%)$ & $12(52.17 \%)$ \\
\hline Enterobacter spp. & 11 & $\begin{array}{l}2 \\
(18.18 \%)\end{array}$ & $\begin{array}{l}8 \\
(72.72 \%)\end{array}$ & $\begin{array}{l}1 \\
(9.10 \%)\end{array}$ \\
\hline Coagulase negative staphylococci (CONS) & 161 & $20(12.42 \%)$ & $139(86.33 \%)$ & $2(1.25 \%)$ \\
\hline Enterococcus spp. & 151 & $74(49.00 \%)$ & $64(42.38 \%)$ & $13(8.62 \%)$ \\
\hline Candida spp. & 133 & $126(94.73 \%)$ & $6(4.51 \%)$ & $1(\% 0.76)$ \\
\hline Staphylococcus aureus & 69 & $5(7.26 \%)$ & $46(66.66 \%)$ & $18(26.08 \%)$ \\
\hline Non-hemolytic Streptococci & 18 & $6(33.33 \%)$ & $11(61.11 \%)$ & $1(5.56 \%)$ \\
\hline Others & 12 & $3(25.00 \%)$ & $8(66.67 \%)$ & 1 (8.33\%) \\
\hline Total & 1280 & $530(41.41 \%)$ & 574 (44.84\%) & 176 (13.75\%) \\
\hline
\end{tabular}

\subsubsection{Gram-positive isolates}

The analysis of the antibiotic susceptibility profile of different gram-positive organisms was conducted and showed that the highest percentage of resistance was exhibited towards penicillin (89.5\%) followed by erythromycin (83.98\%) and then cefoxitin which is the representative of the different beta-lactams in Staphylococcus species (76.52\%). On the other hand, linezolid displayed no resistance $(0 \%)$ and vancomycin resistance was minimal (2.62\%) among gram-positive organisms.

With regards to the predominant resistance phenotypes among different isolates, Staphylococcus aureus (S. aureus) exhibited high resistance rates to many antibiotics where $97.1 \%$ of the isolates displayed resistance to penicillin, and $73.91 \%$ were resistant to gentamicin and all betalactams. On the other hand, S. aureus isolates exhibited a suscepibility of more than $40 \%$ to the rest of antibiotics with $100 \%$ susceptibility to linezolid, nitrofurantoin, and vancomycin where no vancomycin-intermediate $S$. aureus (VISA) or vancomycin-resistant $S$. aureus (VRSA) were found in this study.

Similarly, CONS showed comparable beta-lactam resistance rates to $S$. aureus with a slightly higher level of methicillin resistance (77.6\%), as well as, 100\% susceptibility to linezolid and vancomycin. However, CONS displayed a higher level of resistance to the majority of the rest of the antibiotics.

Enterococci expressed a high level of resistance to both beta-lactams and quinolones, ciprofloxacin (86.75\%), levofloxacin (76.15\%), penicillin (78.14\%), and ampicillin (64.9\%). In a similar pattern to Staphylococci, no linezolid resistance was detected and vancomycin-resistant Enterococci (VRE) were discovered in ten isolates
(6.62\%). Data also revealed a statistically significant difference in the antimicrobial potentials to different isolates. The results of the antibiotic susceptibility profile of each of the Gram-positive pathogens are summarized in Tables 2 and 4 .

\subsubsection{Gram-negative isolates}

Table 3 shows that the least frequency of resistance was recorded against nitrofurantoin (52.5\%), amikacin (58.01\%), followed by imipenem $(59.78 \%)$ and meropenem (61.82\%). Colistin was the most promising antibiotic as all Acinetobacter and Pseudomonas isolates showed $100 \%$ susceptibility to it. Data also revealed that some antimicrobials showed a statistically significant difference in their antimicrobial activities to different bacterial isolates.

Table 3 also shows that among the obtained gramnegative pathogens, Klebsiella was the one that displayed the highest level of multidrug-resistance (87.84\%) followed by Acinetobacter (83.59\%). E. coli and Pseudomonas spp. showed almost identical levels of multidrug-resistance $(73.68 \%, 72.02 \%)$ respectively. Although $70 \%$ or more of the E. coli isolates possessed resistance against the majority of the available antibiotic options, a few agents as amikacin, gentamicin, imipenem, meropenem, and nitrofurantoin showed potential antimicrobial activity with percentage of resistance of $(12.21 \%, 38.37 \%, 18.60 \%, 19.76 \%$, $30.18 \%)$, respectively.

Both Proteus and Enterobacter spp. isolates showed the least antibiotic resistance among gram-negative pathogens. In Enterobacter isolates, the highest level of resistance was recorded against cephalosporins, piperacillin/tazobactam, 
Table 2 Distribution of pathogens associated with intensive care unit-associated infections and their antimicrobial resistance patterns, Ain Shams University Hospitals, Egypt, March 2018-February 2019

\begin{tabular}{|c|c|c|}
\hline Pathogen type and pattern of resistance & $n$ & Resistance, $n$ (\%) \\
\hline Klebsiella spp. & 288 & \\
\hline ESBL production & & $30(10.4)$ \\
\hline Multidrug resistance & & $253(87.84)$ \\
\hline Acinetobacter spp. & 128 & \\
\hline Multidrug resistance & & $107(83.59)$ \\
\hline Pseudomonas spp. & 114 & \\
\hline Multidrug resistance & & $84(73.68)$ \\
\hline Escherichia coli & 172 & \\
\hline ESBL production & & $80(46.5)$ \\
\hline Multidrug resistance & & $124(72.02)$ \\
\hline Proteus spp. & 23 & \\
\hline ESBL production & & $4(17.4)$ \\
\hline Enterobacter spp. & 11 & \\
\hline ESBL production & & $4(36.36)$ \\
\hline Staphylococcus aureus & 69 & \\
\hline MRSA & & $51(73.9)$ \\
\hline Coagulase-negative staphylococci & 161 & \\
\hline MR-CONS & & $125(77.6)$ \\
\hline Enterococcus spp. & 151 & \\
\hline VRE $^{*}$ & & $10(6.6)$ \\
\hline Candida spp. & 133 & \\
\hline Non-hemolytic streptococci & 18 & \\
\hline Others & 12 & \\
\hline Total & 1280 & \\
\hline
\end{tabular}

ESBL extended-spectrum $\beta$-lactamase, MRSA methicillin-resistant $S$ aureus, VRE vancomycin-resistant Enterococcus

*Vancomycin resistance was confirmed by Vitek $2 \mathrm{C}$

doxycycline, and tobramycin in contrast to the rest of antimicrobials which still had considerable activity against Enterobacter. Proteus isolates had the maximum resistance levels observed towards ampicillin/sulbactam (60.08\%), doxycycline $(82.60 \%)$, levofloxacin $(60.86 \%)$, tetracycline (73.91\%), and trimethoprim/sulfamethoxazole (69.56\%). Fortunately, the rest of the antibiotics still had a favorable potential against Proteus.

\section{Discussion}

The excessive use of antibiotics has led to a vast widespread prevalence of antimicrobial resistance. As time passes, bacterial pathogens will defy every antibacterial option, thus, becoming extremely hard to control. Hence, the WHO identified it as an international health prime concern $[12,13]$.

To control this mounting predicament, comprehensive antibiotic stewardship in poor countries is fundamental. However, enough data regarding antimicrobial resistance are unavailable to precisely measure the extent of the problem. The few available studies concerning ICUs suggest that they are hotbeds of emerging high-level resistance. Hence, additional studies in other countries and healthcare settings are encouraged [14].

In this study, gram-negative isolates $(57.5 \%, n=736)$ were more prevalent compared to gram-positive ones $(31.1 \%, n=399)$. Comparable results were found by Halim et al. [15] where gram-negative bacteria took the upper hand among all nosocomial pathogens (53\%) while gram-positive organisms represented $37.9 \%$. Similarly, gram-negative organisms constituted $65.7 \%$ of cases in a study conducted by Sawhney and colleagues [16].

Most of the isolates were recovered from blood cultures $(44.84 \%)$ followed by urine $(41.41 \%)$, unlike the results of Shebl and Mosaad who reported higher recovery from urine specimens in comparison with blood cultures [3].

Among gram-negative organisms, Klebsiella, represented the majority (22.5\%) followed by E. coli (13.4\%). 
Table 3 Antibiotic resistance pattern of the prevalent gram-negative pathogens isolated from patients admitted in ICU, Ain Shams University Hospitals, Egypt, March 2018-February 2019

\begin{tabular}{|c|c|c|c|c|c|c|c|c|}
\hline \multirow[t]{2}{*}{ Antibiotic } & \multicolumn{6}{|c|}{ Resistant isolates, $\boldsymbol{n}(\%)$} & \multirow{2}{*}{$\begin{array}{l}\text { Total } \\
n / N(\%) \\
(N=736)\end{array}$} & \multirow[t]{2}{*}{$p$ value } \\
\hline & $\begin{array}{l}\text { Acinetobacter } \\
\text { spp. } \\
(n=128)\end{array}$ & $\begin{array}{l}\text { Klebsiella } \\
\text { spp } \\
(n=288)\end{array}$ & $\begin{array}{l}\text { E.coli spp. } \\
(n=172)\end{array}$ & $\begin{array}{l}\text { Pseudomonas } \\
\text { spp. } \\
\text { ( } n=114\end{array}$ & $\begin{array}{l}\text { Proteus } \\
\text { spp. } \\
\text { (n=23 }\end{array}$ & $\begin{array}{l}\text { Enterobacter } \\
\text { spp. } \\
(n=11)\end{array}$ & & \\
\hline Amikacin & $113(88.28)$ & $208(72.22)$ & $21(12.21)$ & $76(66.66)$ & $6(26.08)$ & $3(27.27)$ & $\begin{array}{l}427 / 736 \\
(58.01)\end{array}$ & $p<0.001^{*}$ \\
\hline Amoxicillin/clavulanate & ${ }^{* *} \mathrm{NA}$ & 245 (85.06) & $124(72.09)$ & ${ }^{* *} N A$ & $12(52.17)$ & $3(27.27)$ & $\begin{array}{l}384 / 494 \\
(77.73)\end{array}$ & $p<0.001^{*}$ \\
\hline Ampicillin/sulbactam & $110(85.93)$ & 277 (96.18) & $155(90.11)$ & ${ }^{* *} \mathrm{NA}$ & $14(60.08)$ & $4(36.36)$ & $\begin{array}{l}560 / 622 \\
(90.03)\end{array}$ & $p<0.001^{*}$ \\
\hline Cefepime & $110(85.93)$ & $276(96.83)$ & $157(91.27)$ & 91 (79.82) & $7(30.43)$ & $8(72.72)$ & $\begin{array}{l}649 / 736 \\
(88.17)\end{array}$ & $p<0.001^{*}$ \\
\hline Cefotaxime & $124(96.87)$ & 285 (98.95) & $152(88.37)$ & ${ }^{* *} \mathrm{NA}$ & $12(52.17)$ & $10(90.90)$ & $\begin{array}{l}583 / 622 \\
(93.72)\end{array}$ & $p<0.001^{*}$ \\
\hline Cefoxitin & ${ }^{* *} \mathrm{NA}$ & $248(86.11)$ & $92(53.48)$ & ${ }^{* *} \mathrm{NA}$ & $7(30.43)$ & $10(90.90)$ & $\begin{array}{l}357 / 494 \\
(72.26)\end{array}$ & $p<0.001^{*}$ \\
\hline Cefpodoxime & ${ }^{* *} \mathrm{NA}$ & $282(97.91)$ & $160(93.02)$ & ${ }^{* *} N A$ & $10(43.47)$ & $9(81.81)$ & $\begin{array}{l}461 / 494 \\
(93.31)\end{array}$ & $p<0.001^{*}$ \\
\hline Ceftazidime & $115(89.84)$ & $279(96.87)$ & $156(90.69)$ & $92(80.70)$ & $8(34.78)$ & $10(90.90)$ & $\begin{array}{l}660 / 736 \\
(89.67)\end{array}$ & $p<0.001^{*}$ \\
\hline Ceftriaxone & $127(99.21)$ & 285 (98.95) & $152(88.37)$ & ${ }^{* *} \mathrm{NA}$ & $12(52.17)$ & $10(90.90)$ & $\begin{array}{l}586 / 622 \\
(94.21)\end{array}$ & $p<0.001^{*}$ \\
\hline Ciprofloxacin & $111(86.71)$ & $258(89.58)$ & $122(70.93)$ & 91 (79.82) & $13(56.52)$ & $6(54.54)$ & $\begin{array}{l}601 / 736 \\
(81.65)\end{array}$ & $p<0.001^{*}$ \\
\hline Doxycycline & $85(66.41)$ & 235 (81.59) & $123(71.51)$ & ${ }^{* *} \mathrm{NA}$ & $19(82.60)$ & $9(81.81)$ & $\begin{array}{l}471 / 622 \\
(75.72)\end{array}$ & $0.008^{*}$ \\
\hline Gentamicin & 97 (75.78) & $204(70.83)$ & $66(38.37)$ & $81(71.05)$ & $10(43.47)$ & $5(45.45)$ & $\begin{array}{l}463 / 736 \\
(62.90)\end{array}$ & $p<0.001^{*}$ \\
\hline Imipenem & $101(78.91)$ & $225(78.12)$ & $32(18.60)$ & 75 (65.78) & $4(17.39)$ & $3(27.27)$ & $\begin{array}{l}440 / 736 \\
(59.78)\end{array}$ & $p<0.001^{*}$ \\
\hline Levofloxacin & $109(85.15)$ & $231(80.21)$ & $119(69.18)$ & $87(76.31)$ & $14(60.86)$ & $5(45.45)$ & $\begin{array}{l}565 / 736 \\
(76.76)\end{array}$ & $0.001^{*}$ \\
\hline Meropenem & $103(80.46)$ & $228(79.16)$ & $34(19.76)$ & $81(71.05)$ & $4(17.39)$ & $5(45.45)$ & $\begin{array}{l}455 / 736 \\
(61.82)\end{array}$ & $p<0.001^{*}$ \\
\hline $\begin{array}{l}\text { Piperacillin/ } \\
\text { tazobactam }\end{array}$ & 107 (83.59) & $250(86.81)$ & $95(55.23)$ & $80(70.17)$ & $5(21.73)$ & $10(90.90)$ & $\begin{array}{l}547 / 736 \\
(74.32)\end{array}$ & $p<0.001^{*}$ \\
\hline Tetracycline & $114(89.06)$ & $240(83.33)$ & $130(75.58)$ & ${ }^{* *} \mathrm{NA}$ & $17(73.91)$ & $3(27.27)$ & $\begin{array}{l}504 / 622 \\
(81.02)\end{array}$ & $p<0.001^{*}$ \\
\hline Tobramycin & $90(70.31)$ & $234(81.25)$ & $106(61.62)$ & $92(80.70)$ & $6(26.08)$ & $7(63.63)$ & $\begin{array}{l}535 / 736 \\
(72.69)\end{array}$ & $p<0.001^{*}$ \\
\hline $\begin{array}{l}\text { Trimethoprim/ } \\
\text { sulfamethoxazole }\end{array}$ & $87(67.96)$ & 241 (83.68) & $137(79.65)$ & ${ }^{* *} \mathrm{NA}$ & $16(69.56)$ & $3(27.27)$ & $\begin{array}{l}484 / 622 \\
(77.81)\end{array}$ & $p<0.001^{*}$ \\
\hline Colistin & $\begin{array}{l}0 \\
(0)\end{array}$ & ${ }^{* *} \mathrm{NA}$ & ${ }^{* *} \mathrm{NA}$ & $\begin{array}{l}0 \\
(0)\end{array}$ & ${ }^{* *} \mathrm{NA}$ & ${ }^{* *} \mathrm{NA}$ & ${ }^{* *} \mathrm{NA}$ & - \\
\hline Nitrofurantoin & NA & 77 (76.23) & 32 (30.18) & ${ }^{* *} N A$ & ${ }^{* *} \mathrm{NA}$ & $0(0)$ & $\begin{array}{l}109 / 209 \\
(52.15)\end{array}$ & $p<0.001^{*}$ \\
\hline No of urine samples & 33 & 101 & 106 & 49 & 5 & 2 & 296 & \\
\hline
\end{tabular}

${ }^{* *} N A=$ not applicable

* significant at $p<0.05$

On the other hand, CONS (12.5\%) was the most common gram-positive pathogen.

These results were similar to the results reported by other researchers studying bacterial strains in Egypt [15,
17] as well as from countries other than Egypt. Osifo, and Aghahowa from Nigeria reported that E. coli and Klebsiella pneumoniae were the most frequently isolated pathogens [18]. However, others reported a higher level of $E$. 
Table 4 Antibiotic resistance pattern of the prevalent Gram-positive pathogens isolated from patients admitted in ICU, Ain Shams University Hospitals, Egypt, March 2018-February 2019

\begin{tabular}{|c|c|c|c|c|c|}
\hline \multirow[t]{2}{*}{ Antibiotic } & \multicolumn{3}{|l|}{ Resistant isolates, $\boldsymbol{n}$ (\%) } & \multirow{2}{*}{$\begin{array}{l}\text { Total } \\
n / N(\%) \\
(N=381)\end{array}$} & \multirow[t]{2}{*}{$p$ value } \\
\hline & $\begin{array}{l}\text { Staphylococcus aureus } \\
(n=69)\end{array}$ & $\begin{array}{l}\text { Coagulase-negative } \\
\text { staphylococci } \\
(n=161)\end{array}$ & $\begin{array}{l}\text { Enterococcus spp. } \\
(n=151)\end{array}$ & & \\
\hline Amikacin & $\begin{array}{l}34 \\
(49.29)\end{array}$ & $\begin{array}{l}61 \\
(37.88)\end{array}$ & ${ }^{* *} N A$ & $\begin{array}{l}95 / 230 \\
(41.30)\end{array}$ & 0.108 \\
\hline Ampicillin & $\begin{array}{l}\text { NA } \\
* * * 67\end{array}$ & $\begin{array}{l}\mathrm{NA} \\
* * * 156\end{array}$ & $\begin{array}{l}98 \\
(64.90)\end{array}$ & $\begin{array}{l}321 / 381 \\
(84.25)\end{array}$ & - \\
\hline Cefoxitin & $\begin{array}{l}51 \\
(73.91)\end{array}$ & $\begin{array}{l}125 \\
(77.63)\end{array}$ & ${ }^{* *} \mathrm{NA}$ & $\begin{array}{l}176 / 230 \\
(76.52)\end{array}$ & 0.541 \\
\hline Penicillin & $\begin{array}{l}67 \\
(97.10)\end{array}$ & $\begin{array}{l}156 \\
(96.89)\end{array}$ & $\begin{array}{l}118 \\
(78.14)\end{array}$ & $\begin{array}{l}341 / 381 \\
(89.50)\end{array}$ & $p<0.001^{*}$ \\
\hline Clindamycin & $\begin{array}{l}36 \\
(52.17)\end{array}$ & $\begin{array}{l}97 \\
(60.2)\end{array}$ & ${ }^{* *} \mathrm{NA}$ & $\begin{array}{l}133 / 230 \\
(57.82)\end{array}$ & 0.256 \\
\hline Erythromycin & $\begin{array}{l}43 \\
(62.31)\end{array}$ & $\begin{array}{l}130 \\
(80.74)\end{array}$ & $\begin{array}{l}147 \\
(62.31)\end{array}$ & $\begin{array}{l}320 / 381 \\
(83.98)\end{array}$ & $p<0.001^{*}$ \\
\hline Ciprofloxacin & $\begin{array}{l}36 \\
(52.17)\end{array}$ & $\begin{array}{l}107 \\
(66.45)\end{array}$ & $\begin{array}{l}131 \\
(86.75)\end{array}$ & $\begin{array}{l}274 / 381 \\
(71.91)\end{array}$ & $p<0.00^{*} 1$ \\
\hline Doxycycline & $\begin{array}{l}29 \\
(42.02)\end{array}$ & $\begin{array}{l}70 \\
(43.47)\end{array}$ & $\begin{array}{l}38 \\
(25.16)\end{array}$ & $\begin{array}{l}137 / 381 \\
(35.95)\end{array}$ & $0.002^{*}$ \\
\hline Gentamicin (low dose) & $\begin{array}{l}51 \\
(73.91)\end{array}$ & $\begin{array}{l}98 \\
(51.30)\end{array}$ & ${ }^{* *} \mathrm{NA}$ & $\begin{array}{l}149 / 230 \\
(64.78)\end{array}$ & 0.058 \\
\hline Gentamicin (high dose) & ${ }^{* *} N A$ & ${ }^{* *} N A$ & $\begin{array}{l}95 \\
(62.91)\end{array}$ & $\begin{array}{l}95 / 151 \\
(62.91)\end{array}$ & - \\
\hline Linezolid & $\begin{array}{l}0 \\
(0)\end{array}$ & $\begin{array}{l}0 \\
(0)\end{array}$ & $\begin{array}{l}0 \\
(0)\end{array}$ & $\begin{array}{l}0 / 381 \\
(0)\end{array}$ & - \\
\hline Levofloxacin & $\begin{array}{l}33 \\
(47.82)\end{array}$ & $\begin{array}{l}97 \\
(60.24)\end{array}$ & $\begin{array}{l}115 \\
(76.15)\end{array}$ & $\begin{array}{l}245 / 381 \\
(64.30)\end{array}$ & $0.001^{*}$ \\
\hline Teicoplanin & $\begin{array}{l}6 \\
(8.69)\end{array}$ & $\begin{array}{l}25 \\
(15.52)\end{array}$ & $\begin{array}{l}26 \\
(17.21)\end{array}$ & $\begin{array}{l}57 / 381 \\
(14.96)\end{array}$ & 0.250 \\
\hline Tetracycline & $\begin{array}{l}38 \\
(55.07)\end{array}$ & $\begin{array}{l}76 \\
(47.20)\end{array}$ & $\begin{array}{l}53 \\
(35.09)\end{array}$ & $\begin{array}{l}167 / 381 \\
(43.83)\end{array}$ & $0.011^{*}$ \\
\hline Tobramycin & $\begin{array}{l}40 \\
(57.97)\end{array}$ & $\begin{array}{l}117 \\
(72.67)\end{array}$ & ${ }^{* *} N A$ & $\begin{array}{l}157 / 230 \\
(68.26\end{array}$ & $0.028^{*}$ \\
\hline Trimethoprim/sulfamethoxazole & $\begin{array}{l}10 \\
(14.49)\end{array}$ & $\begin{array}{l}100 \\
(62.11)\end{array}$ & ${ }^{* *} N A$ & $\begin{array}{l}110 / 230 \\
(47.82)\end{array}$ & $p<0.001^{*}$ \\
\hline Nitrofurantoin & $\begin{array}{l}0 \\
(0)\end{array}$ & $\begin{array}{l}4 \\
(20.00)\end{array}$ & $\begin{array}{l}38 \\
(28.12)\end{array}$ & $\begin{array}{l}42 / 99 \\
(42.42)\end{array}$ & $0.021^{*}$ \\
\hline Vancomycin & $\begin{array}{l}0 \\
(0)\end{array}$ & $\begin{array}{l}0 \\
(0)\end{array}$ & $\begin{array}{l}10 \\
(6.62)\end{array}$ & $\begin{array}{l}10 / 381 \\
(2.62)\end{array}$ & - \\
\hline No. of urine samples & 5 & 20 & 74 & 99 & \\
\hline
\end{tabular}

***The resistance for ampicillin was deduced from cefoxitin

**NA = not applicable

* significant at $p<0.05$

coli than Klebsiella spp. [3, 19] and S. aureus was much higher than CONS unlike our study [3]. The high prevalence of CONS was justified by Basiri and coworkers, who stated that it could be the overuse of invasive devices with repeated manipulation by healthcare workers and inadequate infection control measures [20].

As regards the distribution of pathogens among the different clinical specimens in the present work, CONS and Klebsiella spp. were the most frequently isolated from blood cultures. Man et al. from Romania also stated that CONS were the most commonly isolated pathogens from blood culture in their study; however, E. coli came before Klebsiella spp. as a cause of bacteremia [21].

In this study, E. coli and Klebsiella spp. were the main pathogens recovered from urine. Similarly, Duffa et al. [22] reported that E. coli and Klebsiella spp. were highly encountered pathogens in urine. As for the predominating pathogens in wound specimens, Klebsiella, Pseudomonas, 
and Acinetobacter spp. were highly recovered organisms in the current research. Ibrahim [23] from Saudi Arabia noticed that most the common wound pathogens were Proteus mirabilis followed by Klebsiella pneumoniae. However, in a research by Magdy and colleagues [24], S. aureus was the most common followed by Pseudomonas aeruginosa, Klebsiella pneumoniae, and E. coli.

The difference between the current study and other studies regarding type and frequency of pathogens could be linked to several factors like environmental conditions, health practices, patient conditions, personal hygiene, number of patients involved in each study, and laboratory procedures [3].

The highest percentage of resistance among grampositive organisms was exhibited towards penicillin (89.5\%) followed by erythromycin (83.98\%) and cefoxitin (76.52\%). Resistance to vancomycin was minimal (2.62\%) among gram-positive organisms while no resistance was noted against linezolid (0\%). Hove et al. [25] reported that the highest rates of resistance were observed against penicillin (90.0\%) and oxacillin (64.0\%). The overall resistance towards penicillin and cefoxitin among staphylococcal isolates of Magdy and colleagues [24] from Egypt was in agreement with the present study. However, their S. aureus isolates displayed higher resistance opposite to the current study where CONS was the species with higher resistance. Moreover, their results were much higher as regards vancomycin resistance which displayed resistance rates of $32.4 \%$ and $41.2 \%$ by $S$. aureus isolates and CONS respectively [24]. Results of Shebl and Mosaad [3] from Egypt were in contrast to the current study as regards vancomycin and linezolid with a resistance of $10.8 \%$ and $11.3 \%$, respectively. However, the results of other researchers regarding vancomycin and linezolid were in favor to that obtained in the present study where Al-Zoubi and his colleagues reported that all their S. aureus isolates were $100 \%$ susceptible to vancomycin [26]. Also, Basak et al. [11] and Mahmoud et al. [27] reported $100 \%$ susceptibility to both vancomycin and linezolid. The higher antibiotic resistance rates reported by the abovementioned researches in comparison to the current results might be attributable to inconvenient use of antimicrobials, geographic and socioeconomic variations, sampling biases, and dissimilar patients' characteristics [28].

As regards Enterococci, the overall resistance pattern was comparable to that reported by Said and Abdelmegeed [29] with the exception of the higher linezolid resistance reported (9.7\%) in their study. Likewise, the resistance pattern of ciprofloxacin, erythromycin, gentamicin, and linezolid agrees with that reported by Zalipour and coworkers [30]. Increased resistance to macrolides and quinolones among enterococcal clinical isolates might be attributed to their massive use for the treatment of enterococcal infections [31, 32].

In the present study, gram-negative isolates exhibited high resistance to almost all the used antibiotic classes with the least frequency recorded against nitrofurantoin (52.5\%), amikacin (58.01\%), followed by imipenem (59.78\%) and meropenem (61.82\%). All isolates of Pseudomonas and Acinetobacter showed 100\% susceptibility to colistin so it can be considered a good therapeutic option [11]. These findings coincide well-with very few exceptions - with those of Eldomany and Abdelaziz [33] from Egypt who reported high antibiotic resistance against their tested isolates of Acinetobacter, Pseudomonas, Klebsiella, Enterobacter, and E. coli from cancer patients.

It is worthy to point to the fact that the high level of resistance observed among the gram-negative organisms in the current study agrees well with the results reported by other researchers [33-35]. Such elevated resistance in Enterobacteriaceae may be attributed to $\beta$-lactamase activity [36].

Generally, the resistance patterns of Acinetobacter and Proteus in the current research were in concordance with Ibrahim [23], although E. coli isolates in Ibrahim's study displayed similar results as regards amikacin, gentamycin, imipenem, and piperacillin/tazobactam but with much lower resistance for the rest of antibiotics. Regarding Pseudomonas and Klebsiella, both showed lower resistance rates in contrast to the present study [23]. Several causes can be responsible for the increased incidence of drug-resistance detected in the present study. The prime reason may be the common practice in Egypt where almost all patients-before hospital admission-take diverse antibiotics either prescribed by doctors or self-medication due to over-the-counter antibiotics administered mostly in an improper dose and for an inadequate period [37, 38]. Other potential causes are the geographical divergence as well as the genetic variations among pathogens from different studies. But unfortunately, data about the molecular characterization of the strains included in the current study are not available.

It is obvious that the obtained results in the current study reflected the high prevalence of multidrug resistance especially among gram-negative pathogens. Klebsiella displayed the highest degree of multidrug-resistance (87.84\%) followed by Acinetobacter (83.59\%). E. coli and Pseudomonas spp. showed almost identical levels of multidrugresistance $(73.68 \%, 72.02 \%)$ respectively, while Proteus and Enterobacter spp. isolates exhibited the lowest resistance among all the gram-negative pathogens.

There is a tremendous increase in MDR gram-negative bacteria in hospitals and especially in the intensive care units (ICU). Such resistance is most notable in ICUs due to the unrestrained usage of antibiotics in ICU in 
comparison to other hospital departments and most of these infections were caused by gram-negative bacilli [39].

It is important to note that the data presented in this study provide a general overview of the current horrifying situation in the hospital under study. This implicates that an action has to be taken to stop this catastrophe by starting an effective action plan for containment.

\section{Conclusions}

The majority of the pathogens in the studied hospital have evolved resistance to most of the antibiotics. This foreshadows an inevitable catastrophe that threatens the future of the medical sector and requires special concern and continuous monitoring.

Conducting more studies to obtain a complete picture of the local and international situation of antibiotic resistance is mandatory in order to guarantee effective antibiotic stewardship before reaching a deadlock with no way out.

\section{Abbreviations}

MDRO: Multidrug-resistant organisms; ASUH: Ain Shams University Hospitals; ICU: Intensive care unit; CDC: The Centers for Disease Control and Prevention; WHO: World Health Organization; CLSI: Clinical Laboratory Standards Institute; ESBL: Extended-spectrum beta-lactamase; MRSA: Methicillin-resistant Staphylococcus aureus; MR-CONS: Methicillinresistant coagulase-negative; VRE: Vancomycin-resistant Enterococci; BHI: Brain heart infusion agar; VISA: Vancomycin-intermediate Staphylococcus aureus; VRSA: Vancomycin-resistant Staphylococcus aureus; S. aureus: Staphylococcus aureus; E.coli: Escherichia coli

\section{Acknowledgements}

None.

\section{Author's contributions}

All of the work in this paper was done by Noha Alaa Eldin Fahim. The author read and approved the final manuscript.

\section{Funding}

This research did not receive any specific grant from funding agencies in the public, commercial, or not-for-profit sectors.

\section{Availability of data and materials}

All data generated or analyzed during this study are included in this published article.

\section{Ethics approval and consent to participate}

This manuscript does not report on or involve the use of any animal or human data or tissue. The research is based on retrospective analysis of data extracted from the laboratory information system. The data was analyzed anonymously and results cannot be traced to individual patients. However, a written permission was obtained from the person in charge of the records. Approval of the Ethical Research Committee, Faculty of Medicine, Ain Shams University was obtained in May 2020.

\section{Consent for publication}

Not applicable.

\section{Competing interests}

The author declares no competing interests.
Received: 7 April 2020 Accepted: 17 December 2020

Published online: 29 March 2021

\section{References}

1. Oduyebo OO, Olayinka AT, Iregbu KC, Versporten A, Goossens H, NwajiobiPrincewill $\mathrm{Pl}$, et al. A point prevalence survey of antimicrobial prescribing in four Nigerian tertiary hospitals. Ann Trop Pathol. 2017;8:42-6. http://www. atpjournal.org/text.asp?2017/8/1/42/224161.

2. Simmons BP, Larson EL. Multiple drug resistant organisms in healthcare: the failure of contact precautions. J Infect Prev. 2015;16(4):178-81.

3. Shebl Rl, Mosaad YO. Frequency and antimicrobial resistance pattern among bacterial clinical isolates recovered from different specimens in Egypt. Cent African J Public Heal. 2019;5(1):36-45.

4. Zhi-Wen Y, Yan-Li Z, Man Y, Wei-Jun F. Clinical treatment of pandrugresistant bacterial infection consulted by clinical pharmacist. Saudi Pharm J. 2015;23(4):377-80

5. Centers for Disease Control and Prevention. Antibiotic resistance threats in the United States. Atlanta: U.S. Department of health and human services, CDC; 2019. https://www.cdc.gov/drugresistance/biggest-threats.html.

6. World Health Organization (WHO). Global action plan on antimicrobial resistance. 2015. http://apps.who.int/iris/handle/10665/193736.

7. Laxminarayan R, Duse A, Wattal C, Zaidi AK, Wertheim HF, Sumpradit N, et al. Antibiotic resistance-the need for global solutions. Lancet Infect Dis. 2013;13(12):1057-98.

8. Tamma PD, Cosgrove SE, Maragakis LL. Combination therapy for treatment of infections with gram-negative bacteria. Clin Microbiol Rev. 2012;25(3): 450-70

9. Clinical and Laboratory Standards Institute. Performance standards for antimicrobial susceptibility testing; twenty-eighth edition (M100). Wayne, PA: Clinical and Laboratory Standards Institute; 2018.

10. Abayneh $M$, Tesfaw $G$, Abdissa A. Isolation of extended-spectrum $\beta$ lactamase- (ESBL-) producing Escherichia coli and Klebsiella pneumoniae from patients with community-onset urinary tract infections in Jimma University Specialized Hospital, Southwest Ethiopia. Can J Infect Dis Med Microbiol. 2018:2018:4846159.

11. Basak S, Singh P, Rajurkar M. Multidrug resistant and extensively drug resistant bacteria: A study. J Pathog. 2016;2016:4065603.

12. World Health Organization (WHO). Antimicrobial resistance: global report on surveillance. 2014

13. Levy SB. The challenge of antibiotic resistance. Sci Am. 1998;278(3):46-53.

14. Bebell LM, Muiru AN. Antibiotic use and emerging resistance: how can resource-limited countries turn the tide? Glob Heart. 2014;9(3):347-58.

15. Halim MMA, Eyada IK, Tongun RM. Prevalence of multidrug drug resistant organisms and hand hygiene compliance in surgical NICU in Cairo University Specialized Pediatric Hospital. Egypt Pediatr Assoc Gaz. 2018;66(4): 103-11.

16. Sawhney N, Shinu P, Singh VA. Bacteriological profile and antibiotic susceptibility pattern of neonatal septicaemia in a tertiary care hospital. Int J Curr Microbiol App Sci. 2015;4(10):977-84

17. Khalifa HO, Soliman AM, Ahmed AM, Shimamoto T, Nariya H, Matsumoto T, et al. High prevalence of antimicrobial resistance in gram-negative bacteria isolated from clinical settings in Egypt: recalling for judicious use of conventional antimicrobials in developing nations. Microb Drug Resist. 2019; 25(3):371-85.

18. Osifo OD, Aghahowa SE. Audit of antibiotic therapy in surgical neonates in a tertiary hospital in Benin City. Nigeria Afr J Paediatr Surg. 2011;8(1):23-8.

19. Beyene D, Bitew A, Fantew S, Mihret A, Evans M. Multidrug-resistant profile and prevalence of extended spectrum $\beta$-lactamase and carbapenemase production in fermentative gram-negative bacilli recovered from patients and specimens referred to National Reference Laboratory, Addis Ababa. Ethiopia PLoS ONE. 2019;14(9):e0222911.

20. Basiri B, Sabzehei MK, Shokouhi M, Moradi A. Evaluating the incidence and risk factors of nosocomial infection in neonates hospitalized in SNICU of Fatemieh hospital in Hamadan, Iran, 2012-2013. Arch Pediatr Infect Dis. 2015;3(2):e23327.

21. Man A, Muntean P, Mare A. Etiology of bacteremic syndromes and bacterial susceptibility of blood culture isolates in a Romanian county hospital. Acta Medica Marisiensis. 2014;60:89-93.

22. Merga Duffa Y, Terfa Kitila K, Mamuye Gebretsadik D, Bitew A. Prevalence and antimicrobial susceptibility of bacterial uropathogens isolated from pediatric patients at Yekatit 12 Hospital Medical College. Addis Ababa 
Ethiopia Int J Microbiol. 2018;2018:8492309. https://doi.org/10.1155/2018/ 8492309.

23. Ibrahim ME. High antimicrobial resistant rates among gram-negative pathogens in intensive care units: a retrospective study at a tertiary care hospital in Southwest Saudi Arabia. Saudi Med J. 2018;39(10): 1035-43.

24. Bahgat MM, Elbialy AA, Zaky MM, Toubar SE. Prevalence of antibiotic resistant aerobic bacteria isolated from surgical wounds of inpatients at Zagazig University Hospitals. Egypt Int J Curr Microbiol App Sci. 2015;4(12): 460-72.

25. Ten Hove R-J, Tesfaye M, Ten Hove WF, Nigussie M. Profiling of antibiotic resistance of bacterial species recovered from routine clinical isolates in Ethiopia. Ann Clin Microbiol Antimicrob. 2017;16(1):46.

26. Al-Zoubi MS, Al-Tayyar IA, Hussein E, Jabali A. Al, Khudairat S. Antimicrobial susceptibility pattern of Staphylococcus aureus isolated from clinical specimens in Northern area of Jordan. Iran J Microbiol. 2015;7(5):265-72.

27. Mahmoud B, EIMasry S, Fahim NAM, Abd ElSattar M, Shaker O. Detection of antibiotic susceptibility by colorimetric minimum inhibitory concentration in staphylococcal isolates. J Appl Microbiol. 2019;127:693-700.

28. Moini AS, Soltani B, Taghavi Ardakani A, Moravveji A, Erami M, Haji Rezaei M, et al. Multidrug-resistant Escherichia coli and Klebsiella pneumoniae isolated from patients in Kashan, Iran. Jundishapur J Microbiol. 2015;25:8(10):e27517.

29. Said HS, Abdelmegeed ES. Emergence of multidrug resistance and extensive drug resistance among enterococcal clinical isolates in Egypt. Infect Drug Resist. 2019;7(12):1113-25.

30. Zalipour M, Esfahani BN, Havaei SA. Phenotypic and genotypic characterization of glycopeptide, aminoglycoside and macrolide resistance among clinical isolates of Enterococcus faecalis: a multicenter based study. BMC Res Notes. 2019;12(1):292.

31. Marosevic D, Kaevska M, Jaglic Z. Resistance to the tetracyclines and macrolide-lincosamide-streptogramin group of antibiotics and its genetic linkage- a review. Ann Agric Environ Med. 2017;24(2):338-44. https://doi. org/10.26444/aaem/74718.

32. Banerjee T, Anupurba S. Risk factors associated with fluoroquinoloneresistant enterococcal urinary tract infections in a tertiary care university hospital in North India. Indian J Med Res. 2016;144(4):604-10. https://doi. org/10.4103/0971-5916.200897.

33. Eldomany R, Abdelaziz N. Characterization and antimicrobial susceptibility of gram-negative bacteria isolated from cancer patients on chemotherapy in Egypt. Arch Clin Microbiol. 2011;2(6):2.

34. Zinner HS. New pathogens in neutropenic patients with cancer: an update for the new millennium. Int J Antimicrob Agents. 2000;16(2):97-101.

35. O'Neill E, Humphreys H, Phillips J, Smyth EG. Third generation cephalosporin resistance among gram-negative bacilli causing meningitis in neurosurgical patients: significant challenges in ensuring effective antibiotic therapy. J Antimicrob Chemother. 2006;57:356-9.

36. Wenzel PR, Sahm FD, Thornsberry C, Draghi DC, Jones EM, Karlowsky JA. In vitro susceptibilities of gram-negative bacteria isolated from hospitalized patients in four European countries, Canada, and the United States in 20002001 to expanded-spectrum cephalosporins and comparator antimicrobials: implications for therapy. Antimicrob Agents Chemother. 2003;47:3089-98.

37. Elden NMK, Nasser HA, Alli A, Mahmoud N, Shawky MA, Ibrahim AAEA, et al. Risk factors of antibiotics self-medication practices among university students in Cairo, Egypt. Open Access Maced J Med Sci. 2020;5;8(E SE-Public Health Education and Training):7-12.

38. Ghazawy ER, Hassan EE, Mohamed ES, Emam SA. Self-medication among adults in Minia, Egypt: a cross sectional community-based study. Health. 2017;9:883-95

39. Azim N, Al-Harbi M, Al-Zaban M, Nofal M, Somily A. Prevalence and antibiotic susceptibility among gram negative bacteria isolated from intensive care units at a tertiary care hospital in Riyadh. Saudi Arabia J Pure Appl Microbiol. 2019;13:201-8.

\section{Publisher's Note}

Springer Nature remains neutral with regard to jurisdictional claims in published maps and institutional affiliations.

\section{Submit your manuscript to a SpringerOpen ${ }^{\circ}$ journal and benefit from:}

- Convenient online submission

- Rigorous peer review

- Open access: articles freely available online

- High visibility within the field

- Retaining the copyright to your article

Submit your next manuscript at $\boldsymbol{\nabla}$ springeropen.com 